\title{
Optimal Solution for the Index Coding Problem Using Network Coding over GF(2)
}

\author{
Jalaluddin Qureshi, Chuan Heng Foh and Jianfei Cai \\ School of Computer Engineering \\ Nanyang Technological University, Singapore \\ jala0001@e.ntu.edu.sg
}

\begin{abstract}
The index coding problem is a fundamental transmission problem which occurs in a wide range of multicast networks. Network coding over a large finite field size has been shown to be a theoretically efficient solution to the index coding problem. However the high computational complexity of packet encoding and decoding over a large finite field size, and its subsequent penalty on encoding and decoding throughput and higher energy cost makes it unsuitable for practical implementation in processor and energy constraint devices like mobile phones and wireless sensors. While network coding over $\mathbf{G F}(2)$ can alleviate these concerns, it comes at a tradeoff cost of degrading throughput performance. To address this tradeoff, we propose a throughput optimal triangular network coding scheme over GF(2). We show that such a coding scheme can supply unlimited number of innovative packets and the decoding involves the simple back substitution. Such a coding scheme provides an efficient solution to the index coding problem and its lower computation and energy cost makes it suitable for practical implementation on devices with limited processing and energy capacity.
\end{abstract}

\section{INTRODUCTION}

The index coding problem [1], [2] is an instance of packets $P=\left\{c_{1}, c_{2}, \ldots, c_{M}\right\}$ transmission problem over a noiseless channel by a transmitter to multiple receivers $R=$ $\left\{R_{1}, R_{2}, \ldots, R_{N}\right\}$, given the subset of packets each receiver already has $H\left(R_{i}\right) \subseteq P$, and the disjoint subset of packets each receiver wants $W\left(R_{i}\right) \subseteq P$, such that the total number of transmissions is minimized, as shown in Fig. 1, It is intuitive to see that the index coding problem can also be extended for erasure channel, where the transmitter solves the index coding problem multiple times until all the receivers have received the packets in their want set. An efficient solution to the index coding problem has several applications, for instance the index coding problem has been shown to occur in content distribution networks [2], satellite communication networks [2], wireless routing [3] and wireless multicasting [4], [5], [6] amongst many other. Network coding [3], [4] has been proposed as an efficient solution for the index coding problem. In network coding, the transmitter generates a coded packet by linearly mapping packets $c_{m} \in P$, with coefficients $g_{m}$, from a finite field $G F\left(2^{q}\right), q \in \mathbb{N}_{1}$, which is then transmitted to the receivers. As we will show later, minimizing the total number of transmissions requires that the transmitted coded packet is linearly independent from the set of packets $H\left(R_{i}\right)$ for the maximum possible number of receivers, ideally for all unsaturated receivers.

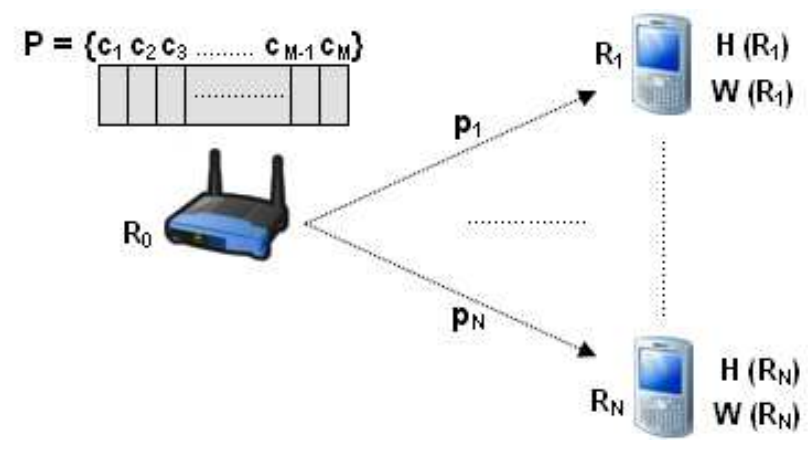

Fig. 1. Application of the index coding problem. Wireless router multicasting a file stream to $N$ receivers.

To illustrate how network coding can be beneficially applicable for the index coding problem, consider for example a wireless network where $R_{0}$ is multicasting packets $c_{1}$ and $c_{2}$ to $R_{1}$ and $R_{2}$. However, $R_{1}$ receives $c_{1}$ but not $c_{2}$, whereas $R_{2}$ receives $c_{2}$ but not $c_{1}$. In this case, rather than retransmitting packet $c_{1}$ and $c_{2}$ in 2 different time slots, it is possible for the transmitter to encode the packets $c_{1} \oplus c_{2}$ over $G F(2)$, and transmit the encoded packet in 1 time slot. On receiving the encoded packet both the receiver can recover the lost packet by decoding the original packet with the encoded packet. This therefore reduces the total number of transmissions and hence improves the network bandwidth.

However the bandwidth performance of network coding for a class of multicast network with a packet batch size of $M$, being transmitted to $N$ receivers, and a Bernoulli packet loss probability of $p_{i}$ for receiver $R_{i}$, has been shown to vary with the size of the finite field $G F\left(2^{q}\right)$ over which packet coding is performed [4], [5]. For random linear network coding (RLNC), where the encoding coefficient is randomly selected from $G F\left(2^{q}\right)$, the probability that the encoded packet $y_{a}$ is linearly independent of all the previously received packets increases logarithmically with respect to $q$ and is bounded by an asymptote [4]. For a deterministic linear network coding (DLNC) scheme where the encoding coefficient $g_{m}$, is selected deterministically by a polynomial-time algorithm, to guarantee that an innovative packe 11 is transmitted at every transmission,

\footnotetext{
${ }^{1}$ An innovative packet with respect to a specified receiver, is defined as a packet which the receiver can't generate from the set of packets it already has.
} 
it has been theoretically shown that the size of the finite field from which the encoding coefficients $g_{m}$, are selected is bounded as $q \geq\left\lceil\log _{2} N\right\rceil$ [5], |7]. Clearly network coding over $G F(2)$ can only guarantee an optimal solution for a network with 2 receivers. It has also been shown that the solution for the index coding problem using network coding over $G F(2)$ is a NP-complete problem [8], [7]. In network coding literature, encoding over $G F(2)$ is referred as XOR coding, whereas encoding over $G F\left(2^{q}\right)$ for $q>1$ is referred to as linear network coding (LNC), we will henceforth use these terms to distinguish between the finite field size from which the coding coefficients are selected.

While LNC over a large field size $G F\left(2^{q}\right)$ has been proposed as a theoretically viable solution to the index coding problem, such benefits do not come without a tradeoff. The most significant drawback of using LNC is the high computational cost of performing packet encoding and decoding [9]. Unlike XOR coding and decoding, where the main mathematical operation is XOR addition, LNC coding involves multiplication and addition whereas LNC decoding involves multiplication and Gaussian elimination. LNC has packet encoding complexity of $\mathcal{O}(M B)$ [6], where $B$ is the length of the data packet. For RLNC the complexity of generating $M$ random coefficients is given as $\mathcal{O}(M G)$, where $G$ is the constant complexity of generating a random number [6]. For DLNC the complexity of deterministically generating $M$ coding coefficients is given as $\mathcal{O}\left(N^{2} M^{3}\right)$ [5]. The decoding computational complexity of decoding $M$ LNC encoded packets is given as $\mathcal{O}\left(M^{2} B+M^{3}\right)$ [6] for both RLNC and DLNC, and hence the decoding complexity per coded packet is given as $\mathcal{O}\left(M B+M^{2}\right)$.

The high energy cost arising because of LNC computational complexity makes LNC unsuitable for practical implementation in battery constrained devices like mobile phones and wireless sensors to solve the index coding problem. Mobile phone batteries, like wireless sensor, suffer from severe energy limitation, and energy optimization for various smartphone applications is becoming an important parameter for designing smartphone applications which can sustain a longer battery life [10], [11]. Using higher capacity battery can't be regarded as a viable alternate approach, as higher power consumption results in the overheating of the devices. Experimental evaluation of RLNC over $G F\left(2^{8}\right)$ for iPod Touch $2 \mathrm{G}$ has shown that packet encoding and decoding can account for up to $33 \%$ of the battery's energy consumption [12]. Whereas it has been shown that XOR-encoding of 2 packets, each 1000 bytes long only consumes $191 \mathrm{~nJ}$ [13] of energy. Given that transmission of a packet of the same length over IEEE 802.11 network on Nokia N95 consumes $2.31 \mathrm{~mJ}$ [13] of energy, the overall energy cost of XOR-coding has no apparent effect on the total energy cost of encoding and transmitting a XOR coded packet. With energy efficiency becoming an increasingly important concern for communication networks [11], even if the solution for the index coding problem using LNC is to be limited for desktop computers and access points (AP), which can afford high energy cost, such an approach would not be green communication feasible because the higher energy cost of LNC corresponds to a higher carbon footprint penalty.

LNC also suffers from low encoding and decoding throughput. The computational penalty cost of LNC on encoding and decoding throughput has also been practically demonstrated on a testbed [14]. In this work the authors show that in general, encoding over $G F(2)$ is approximately 8 times faster than encoding over $G F\left(2^{8}\right)$ on iPhone $3 \mathrm{G}$ implementation. Similarly decoding over $G F(2)$ is approximately 6 times faster than decoding over $G F\left(2^{8}\right)$ on the same testbed.

From the previous discussion it is apparent that a viable solution to the index coding problem is to use a coding scheme which can deliver the bandwidth performance of LNC, while affording the computation cost of XOR coding. To address this gap, in this paper we propose a triangular pattern based packet coding scheme, where packets are encoded over $G F(2)$, and decoding is done using the simple back substitution scheme rather than Gaussian elimination method. Further such coding scheme can guarantee enough 'pool' on linearly independent coded packet to deliver optimal throughput performance. Such optimization tradeoff between energy cost and bandwidth performance has also garnered interest recently [9]. In addition unlike deterministic coding algorithms such as the traditional XOR-coding schemes [3], [15] and DLNC polynomial-time algorithm [5] which require packet feedback information for every transmitted packet from all the receivers, the performance of our proposed coding scheme like RLNC is independent from the constraint of having packet feedback information.

Our paper is organized as follow. We first highlight the current research directions to reduce the energy cost of LNC and increase its encoding and decoding throughput in Section $\amalg$. A formal problem formulation and system parameters are stated in Section IIII We then propose our proposed coding scheme in Section IV] and the evaluation of the triangular coding throughput performance, packet overhead and computational complexity along with comparison with current network coding schemes in Section D Numerical results of the packet overhead of our coding scheme and its comparison with the packet overhead of other network coding scheme are given in Section VI Finally we conclude with the main contributions and results of our paper in Section VII

\section{RELATED WORK}

Current research direction to reduce the computation cost of LNC, and increase the encoding and decoding throughput falls under three broad categories. The first is the use of Gauss-Jordan elimination method running on parallel multiprocessors system as shown in [16], [12], [17] to increase the decoding throughput. This is based on the well-known computer science principle that even though the Gauss-Jordan elimination method requires more computation steps relative to Gaussian elimination, Gauss-Jordan elimination method can nonetheless speedup the processing time required to solve a matrix of fixed size as the number of processors increases [18]. 
This is explained due to the better load balancing characteristics and lower synchronization cost of the Gauss-Jordan elimination method. However such method comes at the tradeoff cost of increasing number of processors requirement, and higher energy cost. Even though both Gaussian elimination and Gauss-Jordan elimination have the same computational complexity order, Gauss-Jordan elimination requires more number of computation steps. A parallel Gauss-Jordan algorithm for multi-processor system is shown to require approximately $50 \%$ more operations than Gaussian elimination [18].

The second major research direction to reduce the decoding computation cost of LNC is to use sparse coding coefficients. A sparse matrix can loosely be described as a matrix with more number of ' 0 's. In [7], [19] it has been shown that when the size of the coding field is bounded as $q \geq\left\lceil\log _{2} N\right\rceil$, there always exists a set of coding coefficient such that the coded packet is linearly independent from the set of packets each of the $N$ receivers have. However, the SPARSITY problem [19] of finding such set of $M$ coding coefficients of which $M-\omega$ coding coefficients are ' 0 ' and the coding coefficient is linearly independent from the set of packets received by all the receivers, is an NP-complete problem with respect to $N$. By assuming fixed $N$, the computational complexity of solving a $M \times M$ matrix of rank $M$ by the receiver reduces from $\mathcal{O}\left(M^{3}\right)$ to $\mathcal{O}\left(M^{2} \omega\right)$, where $\omega \leq M$. Unfortunately such reduction in the complexity of Gaussian elimination come at the tradeoff cost of solving the SPARSITY problem by the transmitter which has complexity given as $\mathcal{O}\left(M^{N}\left(M N^{2}\right)\right)$ [19].

The third major approach to reduce the computation cost is to use the trivial approach of using smaller packet batch size (see [20] and references therein). However such an approach of decreasing the packet batch size comes at the tradeoff cost of decreasing throughput performance [15], [4], [21].

\section{Problem Statement}

Similar to [4], [8], [15] we consider the problem of a wireless transmitter $R_{0}$ multicasting $M$ packets to $N$ receivers. $M$ is called the packet batch size. The iid Bernoulli packet loss at each receiver $R_{i}$ is given as $p_{i}$. At the start of the transmission, $W\left(R_{i}\right)=P, \forall i$. After each transmission, every receiver updates it want and has set, based on the packet it has received. $R_{0}$ continues transmitting packets until $\left|H\left(R_{i}\right)\right|=|P|, \forall i$. Therefore our considered problem of multicasting $M$ packets to $N$ receivers over an erasure channel is a general case of the index coding problem. Let $T$ denote the number of transmissions necessary before all the $N$ receivers receive $M$ innovative packets. The problem statement which we are interested to solve can be written as an optimization problem

$$
\text { minimise } T
$$

subject to

$$
\left|H\left(R_{i}\right)\right|=|P|, \forall i .
$$

In this paper we propose a triangular packet coding scheme which solves (1) optimally, with encoding and decoding computational complexity of the same order as that of traditional XOR packet encoding and decoding.

Therefore the main contribution of our paper is twofold, first we address the energy cost and bandwidth performance tradeoff associated with network coding [9] in this paper. Secondly, from the information theory perspective, we show that unlike previous works which have concluded that the optimal solution for the index coding problem using over $G F(2)$ is NP-complete [7], [5], [8], [15], it is possible to obtain an optimal solution for wireless multicasting using XOR coding when the constraint of adding redundant bits to packets is relaxed.

\section{A. Performance Bound}

The main performance measure for this work is the number of transmissions required to transmit $M$ innovative packets to $N$ memory-based receivers. The performance bound can be derived when an optimal network coding scheme is considered, where every packet transmission is an innovative transmission. Similar to [5], [22], when packet reception is characterized by the binomial probability law, with nonhomogeneous packet loss probabilities, the average number of transmissions required to transmit $M$ innovative packets to $N$ memory-based receivers is

$$
\mathcal{L}=\sum_{n=0}^{\infty}\left\{1-\prod_{j=1}^{N}\left(\sum_{i=M}^{n}\left(\begin{array}{c}
n \\
i
\end{array}\right)\left(1-p_{j}\right)^{i} p_{j}^{n-i}\right)\right\}
$$

where $p_{j}$ is the packet loss probability for receiver $R_{j}$.

We may approximate the above result by only including those whose packet loss are high. With this approximation, the above result can be rewritten as

$$
\mathcal{G}(p, k, M)=\sum_{n=0}^{\infty}\left\{1-\left(\sum_{i=M}^{n}\left(\begin{array}{c}
n \\
i
\end{array}\right)(1-p)^{i} p^{n-i}\right)^{k}\right\},
$$

where $p=\max \left\{p_{i}\right\}$ and $k$ is the number of receivers with packet loss probability $p_{i}=p$.

Axiom 1: For a network with specified $M, k$ and $p$ any network coding scheme which can transmit $\mathcal{G}(p, k, M)$ innovative packets in $\mathcal{G}(p, k, M)$ transmissions is considered an optimal coding scheme.

\section{Proposed Coding Scheme}

We first illustrate the practical usefulness of our proposed coding scheme by the aid of a simple example. Consider the case of $R_{0}$ multicasting packets $c_{1}$ and $c_{2}$ to 4 receivers, $R_{1}, \ldots, R_{4}$. After the first 2 transmissions, only $R_{1}$ receives $c_{1}$ and only $R_{2}$ receives $c_{2}$. Now to transmit a packet which is innovative for all the receivers, the only possibility is to transmit $c_{1} \oplus c_{2}$, when coding is limited to $G F(2)$. However let us assume that only $R_{3}$ receives this coded packet. Given the constraint of coding over $G F(2)$ it is easy to verify that after these 3 transmissions, there is no possibility to transmit a packet which will be innovative for all the receivers. This 
is also consistent with previous theoretical analysis [5], [7], which have proven that an innovative packet transmission, for every transmission is only possible on the condition that the field size is larger than or equal to the number of users.

In our proposed coding scheme we go around this information theory limitation, by adding redundant bits to $c_{1}$ and $c_{2}$, then encoding these packets over $G F(2)$, and including information about these redundant bits added to each packet in the packet header of the encoded packet. For the given example, we add bit ' 0 ' at the head of data payload of packet $c_{1}$. To equalize the length of both the packets we add bit ' 0 ' at the tail of the data payload of packet $c_{2}$, and then encode these packet over $G F(2)$. Let us assume that all packets $c_{m} \in P$, $1 \leq m \leq M$, have equal data payload length of $B$ bits. The binary bit pattern of the data payload for packet $c_{m}$ can be represented as $\left(b_{1, m}, b_{2, m}, \ldots, b_{B, m}\right), b_{j, m} \in\{0,1\}$. Hence the bit pattern of $c_{1}$ with one redundant bit added at the head of the packet is given as $\left(0, b_{1,1}, \ldots, b_{B, 1}\right)$, and that of $c_{2}$ as $\left(b_{1,2}, \ldots, b_{B, 2}, 0\right)$. We denote such modified packet as $c_{m, r_{m}}$, where $r_{m} \in \mathbb{N}_{0}$, is the number of ' 0 's added at the head of the data payload of packet $c_{m}$. The new encoded packet is denoted as $c_{1,1} \oplus c_{2,0}$. The packet header will include information about $r_{m}$ for each packet used for encoding, and we will study the overhead cost in subsequent section.

This new encoded packet $c_{1,1} \oplus c_{2,0}$ will be innovative for all the 4 receivers. If $R_{3}$ receives this packet, it will have the information about bit $b_{1,2}$ as bit ' 0 ' was added as a redundant bit in packet $c_{1}$ from the packet header. Since $R_{3}$ also has packet $c_{1} \oplus c_{2}$, it now has information about bit $b_{1,2}$ and $b_{1,1} \oplus$ $b_{1,2}$. Using this information it can decode bit $b_{1,1}$. Bit $b_{1,1}$ is then substituted in $b_{1,1} \oplus b_{2,2}$, from the encoded packet $c_{1,1} \oplus c_{2,0}$, to obtain bit $b_{2,2}$. Therefore using this bit-by-bit simple back substitution method, $R_{3}$ can decode all the bits of packet $c_{1}$ and $c_{2}$.

\section{A. Generation of Innovative Packets}

For a system such the total number of redundant bits added is $r_{\max }$ to a packet of length $B$ bits, then we note that generating $c_{m, r_{m}}$ by adding of redundant bits to $c_{m}$ is equivalent to the following operation

$$
c_{m, r_{m}}=2^{r_{\max }-r_{m}} c_{m} .
$$

An encoded packet $c_{1, r_{1}} \oplus c_{2, r_{2}} \oplus \cdots \oplus c_{M, r_{M}}$ is said to be innovative to a receiver if the encoded packet is linearly independent with respect to all other encoded packets that the receiver already possesses. Our aim is find a sequence of coefficient sets such that an encoded packet with a coefficient set is always linearly independent to encoded packets of any collection of other coefficient sets, and secondly, the decoding procedure can be solved using the back substitution method.

Assuming encoding of $M$ packets, for an encoded packet $y_{a}=c_{1, r_{1}} \oplus c_{2, r_{2}} \oplus \ldots \oplus c_{M, r_{M}}$, the total number of redundant bits added to each packet $c_{m}$ is $r_{\max }=$ $\max \left\{r_{1}, r_{2}, \ldots, r_{M}\right\}$. We represent the unique id of encoded packet $y_{a}$ as $\left(r_{1}, r_{2}, \ldots, r_{M}\right)_{a}$. The encoded packet can be written as

$$
2^{r_{\max }-r_{1}} c_{1} \oplus 2^{r_{\max }-r_{2}} c_{2} \oplus \ldots \oplus 2^{r_{\max }-r_{m}} c_{m} .
$$

We shall propose a natural number based triangular substitution based network coding. The Gaussian elimination method consists of 2 major steps, the matrix triangularization step and the back substitution step. The matrix triangularization step accounts for $\mathcal{O}\left(M^{3}\right)$ steps, while the back substitution step requires only $\mathcal{O}\left(M^{2}\right)$ steps [23]. Therefore our aim is to design a coding scheme such that the receiver does not have to perform the triangularization step. In other words, with our proposed coding scheme, the encoding packets are linearly independent and back substitution ready.

If packet encoding is performed such that for an encoded packet, $y_{a}$ with an id of $\left(r_{1}, r_{2}, \ldots, r_{M}\right)_{a}, r_{m}$ is mapped to an element of the set of natural number sequence given as $\{0,1, \ldots,(M-1)\}$ using a bijective function, then a collection of encoded packets can easily form a triangular augmented matrix. Without loss of generality, assume that the first encoded packet (also called the initial pattern) has an id given as $(0,1,2,3, \ldots, M-1)_{1}$. Then to generate the next encoded packet, we anchor ' 0 ' at its position and rotate all other terms rightward which results in $(0, M-1,1,2, \ldots, M-2)_{2}$. Similarly the consecutive encoded packets in the series are generated as $(0, M-2, M-1,1, \ldots, M-3)_{3},(0, M-3, M-$ $2, M-1, \ldots, M-4)_{4}, \ldots,(0,2,3,4, \ldots, M-1,1)_{M-1}$. We call all encoded packets in this series, with the ' 0 ' anchored in one position, to form a group. We may now anchor ' 0 ' at the second position starting with an id $(1,0,2,3, \ldots, M-1)_{M}$ for the next group. By rotating all other terms except ' 0 ', we get another group of $M-1$ encoded packets. Given $M$ positions for ' 0 ' to anchor, we yield altogether $M$ groups of $M-1$ encoded packets with the very last encoded packet being $(2,3,4, \ldots, M-1,1,0)_{M(M-1)}$.

But what if the transmitter needs to generate additional encoded packets? In such case, after the $M(M-1)$ permutations has been exhausted, the transmitter may start with another initial pattern of $(0,2,4,6, \ldots, 2 M-2)_{M(M-1)+1}$ which is derived from the earlier initial pattern with each $r_{m}$ multiplied by a constant $\alpha$. Let $\alpha=2$, and we call this collection of encoded packets the second round. Similarly, the transmitter can generate another $M(M-1)$ encoded packets for the third round by setting $\alpha=3$. Theoretically, we may continue with $\alpha=3,4,5, \ldots$ without a limit, only that a higher value of $\alpha$ results in a larger value of $r_{\max }$ which implies more redundant bits for the encoding.

In the following, we shall show that our proposed coding scheme always generate innovative packets. In other words, the coefficient matrix of any given $n$ encoded packets where $n \leq$ $M$ gives a rank of $n$. While our proposed coding scheme mixes standard arithmetic (i.e. multiplication by shifting) and finite field arithmetic (i.e. exclusive-or), use of standard arithmetic is sufficient to prove that every generated packet is innovative.

Lemma 1: Consider a system of $M$ packets. Given an encoded packet $y_{a}$ with an id of $(0,1,2,3, \ldots, M-1)_{a}$, the 
coefficient matrix formed by all $M-1$ encoded packets in the same group of $y_{a}$ including $y_{a}$ gives a rank of $M-1$.

Proof: We first list all the ids of $M-1$ encoded packets in the same group of $y_{a}$ including $y_{a}$ as follows where

$$
\begin{array}{ccccc}
(0, & 1, & 2, & 3, \ldots, & M-1)_{a} \\
(0, & M-1, & 1, & 2, \ldots, & M-2)_{a+1} \\
(0, & M-2, & M-1, & 1, \ldots, & M-3)_{a+2} \\
& \vdots & & \\
(0, & 2, & 3, & 4, \ldots, & 1)_{a+M-2} .
\end{array}
$$

From the id of $y_{a}$, we know that $r_{\max }=M-1$ and $\alpha=1$. The above list of encoded packets can be expressed by

$$
\begin{array}{rrrrrrr}
2^{M-1} c_{1} & + & 2^{M-2} c_{2} & + & 2^{M-3} c_{3} & +\cdots & +2^{0} c_{M} \\
2^{M-1} c_{1} & + & 2^{0} c_{2} & + & 2^{M-2} c_{3} & +\cdots & +2^{1} c_{M} \\
2^{M-1} c_{1} & + & 2^{1} c_{2} & + & 2^{0} c_{3} & +\cdots & +2^{2} c_{M} \\
\vdots & \ddots & \vdots & & & & \\
2^{M-1} c_{1} & + & 2^{M-3} c_{2} & + & 2^{M-4} c_{3} & +\cdots & +2^{M-2} c_{M} .
\end{array}
$$

For each pair of consecutive equations above, we multiply the upper one by $2^{\alpha}$ and then minus with the lower one. After this simplification, we get

$$
\begin{array}{rrr}
2^{M-1} c_{1} & + & \left(2^{M-1}-1\right) c_{2} \\
2^{M-1} c_{1} & + & \left(2^{M-1}-1\right) c_{3} \\
2^{M-1} c_{1} & + & \left(2^{M-1}-1\right) c_{4} \\
& \vdots & \\
2^{M-1} c_{1} & + & \left(2^{M-1}-1\right) c_{M}
\end{array}
$$

which clearly gives a rank of $M-1$.

For other groups, we may reorder the coefficients such that $r_{1}=0$ and then repeat Lemma 1 to show that other groups possess the same property. Furthermore, Lemma 1 can be easily extended to the case of $\alpha=2,3, \ldots$ which applies to a group belonging to higher rounds.

Lemma 2: Consider a system of $M$ packets. A collection of all $M-1$ encoded packets in the same group and an encoded packet from a different group gives a rank of $M$.

Proof: We shall prove by calculating the determinant of the matrix. Without loss of generality, we consider a group, $\mathbf{G}$, of encoded packets starting with the initial pattern of $(0,1,2,3, \ldots, M-1)_{1}$. Based on Lemma 1 after simplification, these encoded packets can be represented by

$$
\begin{array}{rrr}
2^{M-1} c_{1} & + & \left(2^{M-1}-1\right) c_{M} \\
2^{M-1} c_{1} & + & \left(2^{M-1}-1\right) c_{M-1} \\
2^{M-1} c_{1} & + & \left(2^{M-1}-1\right) c_{M-2} \\
& \vdots & \\
2^{M-1} c_{1}+ & \left(2^{M-1}-1\right) c_{2}
\end{array}
$$

Let us consider an arbitrary encoded packet, $y_{a}$, with the following representation

$$
y_{a}=\lambda_{1} c_{1}+\lambda_{2} c_{2}+\lambda_{3} c_{3}+\cdots+\lambda_{M} c_{M} .
$$

Combining the last two results, we form a coefficient matrix and express its determinant, $d$ as

$$
\left|\begin{array}{ccccc}
2^{M-1} & 0 & 0 & \ldots & 2^{M-1}-1 \\
& & \vdots & & \\
2^{M-1} & 0 & 2^{M-1}-1 & \ldots & 0 \\
2^{M-1} & 2^{M-1}-1 & 0 & \ldots & 0 \\
\lambda_{1} & \lambda_{2} & \lambda_{3} & \ldots & \lambda_{M}
\end{array}\right| .
$$

The condition for $y_{a}$ to be linearly independent of the encoded packets from group $\mathbf{G}$ is that $d$ produces a none zero value. After some algebraic manipulation on $d$ and applying the condition where $d \neq 0$, we yield the following condition where

$$
2^{M-1} \sum_{i=2}^{M} \lambda_{i} \neq\left(2^{M-1}-1\right) \lambda_{1} .
$$

Our coding scheme demands the one-to-one mapping between $\left\{r_{1}, r_{2}, \ldots, r_{M}\right\}$ and $\{0,1, \ldots,(M-1)\}$. In other words, $\left\{\lambda_{1}, \lambda_{2}, \ldots, \lambda_{M}\right\}$ are mapped one-to-one to $\left\{2^{(M-1)}, 2^{(M-2)}, \ldots, 2^{0}\right\}$. The only case that (3) cannot be met occurs when $\lambda_{1}=2^{(M-1)}$. In other cases, we always have

$$
\sum_{i=2}^{M} \lambda_{i}>\lambda_{1}>0
$$

and since $2^{M-1}>\left(2^{M-1}-1\right)>0$, condition (3) is always met.

The setting of $\lambda_{1} \neq 2^{(M-1)}$ requires that $r_{1} \neq 0$ for $y_{a}$ of id $\left(r_{1}, r_{2}, \ldots, r_{M}\right)$. As $y_{a}$ is drawn from a group other than group $\mathbf{G}$, its ' 0 ' is anchored at a different position rather than at $r_{1}$ like those in group $\mathbf{G}$, thus we have $r_{1} \neq 0$, and hence $y_{a}$ is linearly independent of the encoded packets from group G.

Applying a quick test using (3) in Lemma 2 shows that based on our coding scheme, a pick of an encoded packet even from a group of other rounds satisfies the condition. Consequently, with the above lemmas, we established that every encoded packet is an innovative packet.

\section{B. Packet Decoding}

We now show that such a coding scheme can be solved by substitution method. We illustrate the concept using an example depicted in Fig. 2. In our example, we consider a set of 4 original packets, that is $M=4$. After transmissions of several encoded packets, we consider that a receiver has successfully collected 4 encoded packets, $y_{1}, y_{2}, y_{3}$ and $y_{4}$ with the packet ids of $(0,1,2,3)_{1},(1,0,2,3)_{2},(3,0,1,2)_{3}$ and $(1,2,3,0)_{4}$ respectively. In Figure 2, each table represents an encoded packet where each row lists the bits of an original packet involved in the encoding. It can be seen that the first bit of $y_{1}$ counting from the left is encoded by $b_{1,1} \oplus 0 \oplus 0 \oplus 0$ which equals $b_{1,1}$. Similarly, $b_{1,2}$ and $b_{1,4}$ can be obtained from the first bits of $y_{2}$ and $y_{4}$ respectively. Now, the decoder may proceed to the second bit position of the 4 packets. Substituting $b_{1,1}, b_{1,2}$ and $b_{1,4}$ into the $(M-1)$ matrices, $b_{2,1}$, $b_{2,2}, b_{2,4}$ and $b_{1,3}$ can be obtained immediately. Moving on to 


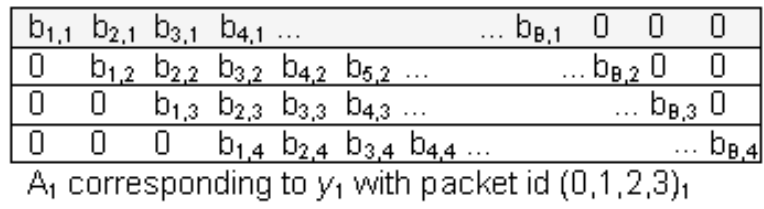

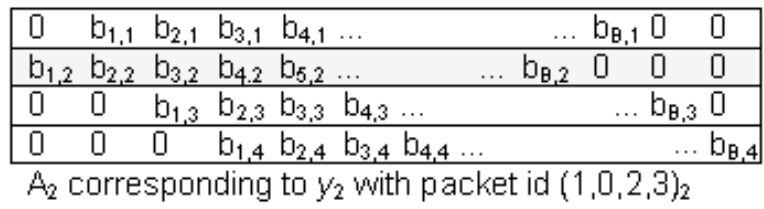

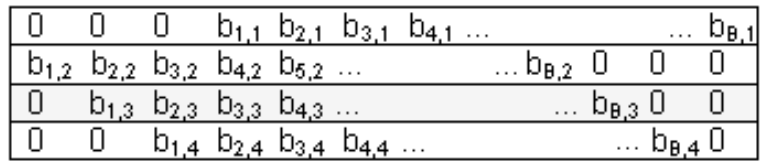

$\mathrm{A}_{3}$ corresponding to $y_{3}$ with packet id $(3,0,1,2)_{3}$

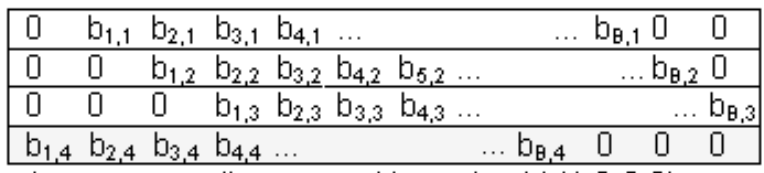

$\mathrm{A}_{4}$ corresponding to $y_{4}$ with packet id $(1,2,3,0)_{4}$

Fig. 2. An illustrating example to show how packet decoding is done on triangular network coding scheme. Highlighted $b_{j, m}$ represent calculated bit value from an equation with only 1 unknown variable, whereas nonhighlighted $b_{j, m}$ represent substituted bit value. Bits ' 0 ' are known bit values from the packet's header. Packet $y_{2}$ and $y_{3}$ are from the same group.

the bit position, bits $b_{3,1}, b_{3,2}, b_{2,3}$ and $b_{3,4}$ can be immediately solved by substitution. The process can continue further until all unknown bits are solved. By this way, a receiver can decode the bits of all 4 packets through the simple back substitution at the bit level.

\section{Performance Evaluation}

\section{A. Throughput Performance}

Based on Axiom 1, if for a network with specified $M$, $k$ and $p$, our proposed coding scheme can generate at least $\mathcal{G}(p, k, M)$ innovative packets, then it can be considered as an optimal coding scheme. It is easy to verify that for

$$
\alpha\left(M^{2}-M\right) \geq \mathcal{G}(p, k, M)
$$

there always $\exists \alpha$ such that the above inequality is satisfied. As we will show later in Section VI for most practical setting (generally corresponding to $M \geq 10$ ) $\alpha=1$ is sufficient to guarantee at least $\mathcal{G}(p, k, M)$ innovative packets. For smaller $M$, a small value of $\alpha$ is sufficient, and therefore our proposed coding scheme does not have significantly high packet overhead. In practice, a very small value of $M$ is often not desirable as it does not provide sufficient pool of packets to utilize the benefit of network coding. We formally study the packet overhead cost of our proposed coding scheme in subsequent subsection.
The throughput performance of DLNC is optimal when $q \geq\left\lceil\log _{2} N\right\rceil$. However in the paper [5], the optimal bound and DGC (Dynamic General Coding) 2 throughput performance results do not match. This is explained as follow. The throughput bound the authors consider is not tight, however since the DGC algorithm claims to be able to always find coding coefficients which is linearly independent for all the receivers, implies that the DGC is an optimal coding scheme.

Assuming fixed $N$, sparse LNC can be solved in polynomial time to generate sparse and linearly independent coding coefficients, otherwise optimal throughput for sparse LNC is NP-complete. However since solving SPARSITY for a fixed network size is a special case of the SPARSITY problem, therefore in the general sense the throughput optimality of sparse LNC is considered NP-complete.

\section{B. Packet Overhead}

For LNC, the packet header needs to include information about encoding coefficient $g_{m}$ used to multiply with $c_{m}$. If $g_{m} \in G F\left(2^{q}\right)$, the number of bits required to represent $g_{m}$ is given as $\log _{2} 2^{q}$. Since there are $M$ such encoding coefficients, the total packet overhead of RLNC is given as $M \log _{2} 2^{q}$, which reduces to $M q$ bits. For an optimal DLNC and sparse coding, $q$ is bounded by $q \geq\left\lceil\log _{2} N\right\rceil$ [5], [19], therefore the total packet overhead for an optimal DLNC and sparse coding scheme is given as $M\left\lceil\log _{2} N\right\rceil$.

For our proposed coding scheme, the packet overhead is given by $r_{\max }$ redundant bits added to every packet, and $M\left\lceil\log _{2} r_{\max }\right\rceil$ bits to store the encoded packet unique id $\left(r_{1}, \ldots, r_{M}\right)_{a}$ in the encoded packet header. Therefore the total packet overhead of our proposed coding scheme is given as $r_{\max }+M\left\lceil\log _{2} r_{\max }\right\rceil$ bits, where $r_{\max }=\alpha(M-1)$, and $\alpha$ corresponds to the smallest value required to satisfy inequality (4). For $\alpha=1$ (which satisfies inequality (4) when $M$ is reasonably large), the total packet overhead is $M-1+M\left\lceil\log _{2}(M-1)\right\rceil$ or simply $M+M\left\lceil\log _{2} M\right\rceil$ if we ignore the insignificant constant term of minus one.

We would like to further point out that the packet overhead of triangular network coding can be reduced to approximately $M$ bits, which is the number of redundant bits added to each packet. The only information which needs to be included in the packet header is the packet batch size, the group index and packet index. Since triangular network coded packets' id follow a sequence, with these information in the packet header, the receiver can reconstruct the packet id of the coded packet.

However for our coding comparison we assume the packet overhead of $M+M\left\lceil\log _{2} M\right\rceil$ for triangular network coding to provide fair comparison with RLNC packet overhead, since assuming that both the transmitter and receiver have the same random number generator (RNG), all what a RLNC coded packet then needs to include in the packet header is the seed and packet index. Using the seed and packet index, the receiver can then regenerate the random coding coefficients.

\footnotetext{
${ }^{2}$ DGC is the name of a DLNC based algorithm demonstrated in [5].
} 


\section{Computational Complexity}

We evaluate three distinct computational complexity. The algorithm complexity refers to the complexity of generating the coding coefficients for $M$ coded packets. The total algorithm complexity is given as $M$ times the complexity of finding $M$ coding coefficient for each coded packet. The encoding complexity refers to the complexity of encoding $M$ coded packets. Whereas the decoding complexity signifies the complexity of decoding $M$ innovative coded packets by a receiver.

We evaluate the computational complexity for $M$ coded packets for uniformity in comparison, though in practise the total algorithm and encoding complexity would also be dependent on the throughput optimality of the coding scheme. Optimal coding scheme will need to run the algorithm and perform coding $\mathcal{G}(p, k, M)$ times only, whereas suboptimal coding schemes will need to run the algorithm and perform coding $\mathcal{G}(p, k, M)+\mathcal{C}$ times, where $\mathcal{C}$ is a coding scheme dependent arbitrary constant.

The decoding complexity is more significant than the algorithm and encoding complexity. This is because the algorithm and encoding computation cost is borne by a single transmitter which is often not a battery constrained device such as an AP, whereas the decoding cost is borne by $N$ receivers, which are often battery and processor constrained such as smartphones.

We evaluate the encoding and decoding complexity at bit level, so that this way we can distinguish between the complexity of multiplication used in LNC and addition used in XOR coding. For two non-negative integers $a$ and $b$, the multiplication and addition complexity is given as $\mathcal{O}\left(\left(\log _{2} a\right)\left(\log _{2} b\right)\right)$ and $\mathcal{O}\left(\log _{2} a+\log _{2} b\right)$ respectively [24]. While there exist different optimization algorithms to reduce the computational complexity of multiplication, such optimization survey is beyond the scope of the paper.

1) Traditional XOR: The encoding complexity of a traditional XOR packet encoding of $m$ packet 3 is given as $\mathcal{O}(m B)$, where $m \leq M$. XOR encoding is performed bitby-bit. For each bit $\left(b_{j, m}\right)$ position it needs to evaluate the value of $m$ bits in location $j$. Therefore the average computational complexity to generate $M$ coded packets is given as $\mathcal{O}(M m B)$. Similarly XOR-encoded packet can be decoded by performing the XOR operation on a set of packets, which has complexity given as $\mathcal{O}(m B)$. To decode $M$ encoded packets, the average computational complexity is given as $\mathcal{O}(M m B)$.

For the algorithm complexity we consider the sort-byutility algorithm [15] for our evaluation, which has complexity given as $\mathcal{O}\left(M^{2} \log _{2} M\right)$. We have chosen the sort-by-utility algorithm, as it has been shown to be an efficient XOR coding algorithm with respect to other known XOR coding algorithms in [15].

2) $R L N C:$ RLNC needs to perform random number generation, multiplication and addition during encoding. Generating a random number has constant time complexity of $G$. Therefore

\footnotetext{
${ }^{3}$ XOR coding is often performed on a subset of packets from $P$ [3], [15, where $|P|=M$, the cardinality of the subset of packets over which encoding is performed varies for each encoded packet and is algorithm dependent.
}

the total algorithm complexity is given as $\mathcal{O}\left(M^{2} G\right)$. The operation $g_{m} \cdot c_{m}$ has complexity given as $\mathcal{O}(q B)$. To generate a coded packet $M$ such operations needs to be performed, hence the complexity to generate a coded packet is given as $\mathcal{O}(M q B)$. Therefore the total encoding complexity to generate $M$ coded packets is given as $\mathcal{O}\left(M^{2} q B\right)$.

For decoding, RLNC needs to perform Gaussian elimination with complexity given as $\mathcal{O}\left(M^{3}\right)$, and then multiplication and addition of $M$ coded packet with the inverse matrix. Therefore the total decoding complexity is given as $\mathcal{O}\left(M^{2} q B+M^{3}\right)$.

3) DLNC: The only known DLNC algorithm is the DGC algorithm given in [5]. DGC has algorithm complexity given as $\mathcal{O}\left(N^{2} M^{3}\right)$ for one coded packet. Its encoding and decoding complexity is the same as that of RLNC.

4) Sparse LNC: Sparse LNC is a special case of DLNC. Unlike DLNC, where the main objective is to find coding coefficients which are linearly independent from received coding coefficients by all the receivers, in sparse LNC, the objective is not only to find linearly independent coding coefficients, but also coefficients which are sparse. The algorithm complexity for sparse LNC is given as $\mathcal{O}\left(M^{N}\left(M N^{2}\right)\right)$, which reduces to $\mathcal{O}\left(M^{N} N^{2}\right)$. Since there are large number of ' 0 ' coding coefficients in sparse LNC we can ignore the computational cost of $g_{m} \cdot c_{m}$ when $g_{m}=0$. Hence the coding coefficient is given as $\mathcal{O}(M q \omega B)$, where $\omega$ is the number of non-zero components, $\omega \leq M$. The decoding complexity is given as $\mathcal{O}\left(M^{2} q B+\omega M^{2}\right)$.

5) Triangular Network Coding: The number of flops (or number of steps) for our proposed triangular network coding scheme is given as follow. Generation of unique packet ids for packet encoding has constant time complexity, as these packet id follow a natural number based sequence. The algorithm to generate the coefficient matrix for $M$ packets has complexity of $\mathcal{O}\left(M^{2}\right)$. For encoding complexity, the encoding of each packet has complexity of $\mathcal{O}(M B)$, since there are $B$ bits from $M$ packets, and XOR addition is the only required operation. The redundant ' 0 ' bits can be ignored both during encoding and decoding. Therefore to generate $M$ such encoded packet, the overall complexity is given by $\mathcal{O}\left(M^{2} B\right)$.

Once a receiver has $M$ innovative encoded packets, then the complexity to decode these $M$ packets is given as $\mathcal{O}\left(M^{2} B\right)$. We elaborate on the back-substitution process. $M$ flops are required to find the solution of each bit (XOR addition), the solution for this equation is then substituted in $M-1$ matrices. Since there are $M$ such equations the total number of flops is given as $M(2 M-1)$. Hence the decoding computational complexity for each bit location is given as $\mathcal{O}\left(M^{2}\right)$, which is also consistent with the results shown in [23].

Our proposed coding scheme results in triangular pattern both at the start and end of the packet (See Figure 2 for illustration), and therefore for a multiprocessor system, it is possible that one processor decodes the first half part of the packets from the start, while simultaneously another processor decodes the second half part from the end of the packet (reverse direction) using the substitution method. This can cut down the decoding time by half in a multiprocessor system. 


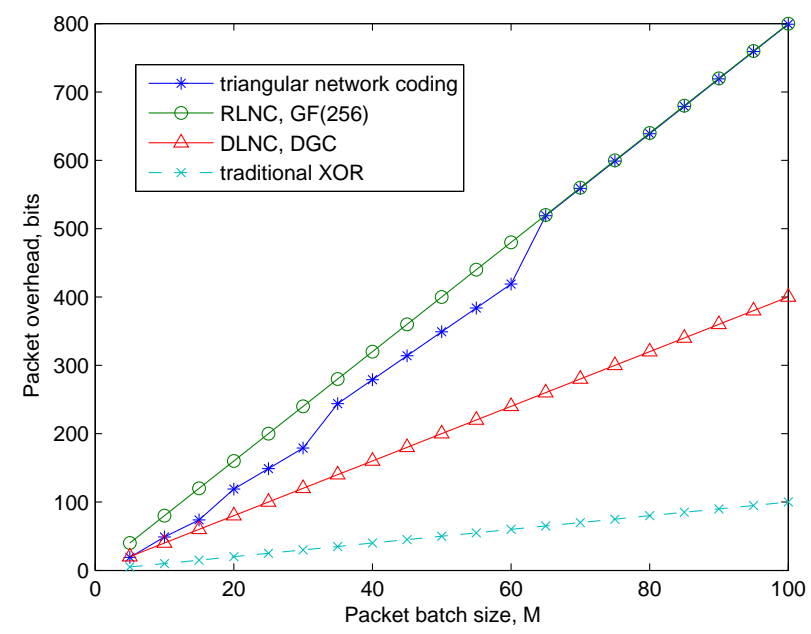

Fig. 3. Packet overhead cost for $\mathrm{N}=10$ and $\mathrm{p}=0.3$.

\section{Packet Feedback Requirement}

We further note that traditional XOR, DLNC and sparse $\mathrm{LNC}$ require feedback information from all receivers to decide what coefficient sets to be used for further encoding of packets such that the encoded packets can be innovative to all receivers. In this aspect, RLNC and our proposed scheme do not need the feedback information. For RLNC, it simply generates encoded packets using random numbers. For our proposed scheme, the transmitter can simply continue the sequence of coefficient sets as the generated encoded packets will always be innovative.

Collecting packet feedback from $N$ receivers over an erasure channel induces a very large overhead. For wireless networks, the IEEE 802.11-2007 standard does not provide any MAC level reliability for multicast transmissions. Therefore deterministic network coding schemes are often based on an impractical arbitrary assumption that the transmitter has perfect feedback information for all transmitted packets.

\section{NumericAl RESULTS}

We now study the packet overhead corresponding to the smallest $\alpha$ necessary to satisfy inequality (4), and compare the results with the packet overhead of RLNC. Without loss of generality, we assume that $p_{1}=\ldots=p_{N}=p$, such that $k=N$. Unlike the packet overhead of triangular coding, which is a monotonic function with respect to $M$, RLNC and DLNC packet overhead cost are a function of two variables.

For RLNC there is no standardized field size suggested, though an overwhelming majority of works have considered a field size of $G F\left(2^{8}\right)$ for RLNC as being sufficiently large enough to guarantee linear independency with very high probability [12], [9], [14], [4]. We therefore assume $q=8$ for the evaluation of RLNC packet overhead.

The result of packet overhead for various coding scheme for different $M, N$, and $p$ is given in Figs. 3 and 4 . The results show that for practical setting, the packet overhead cost of our

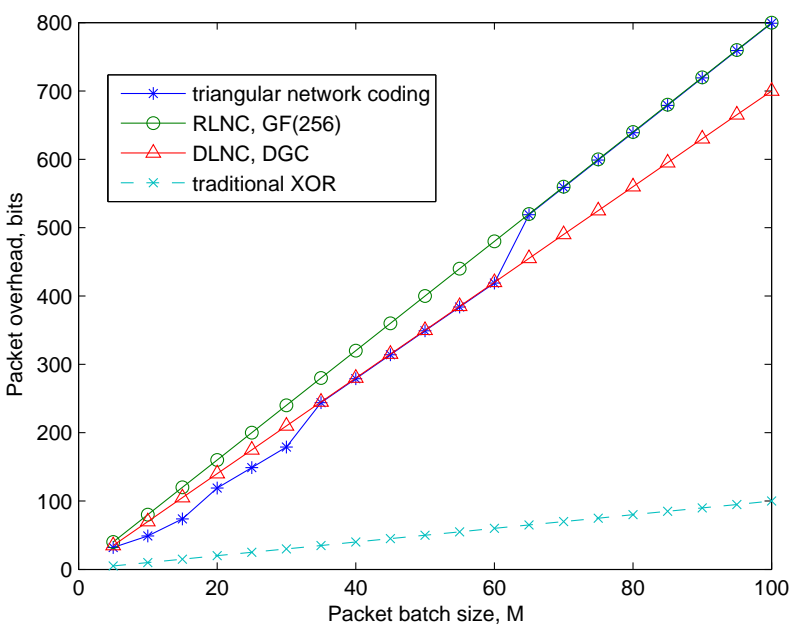

Fig. 4. Packet overhead cost for $\mathrm{N}=100$ and $\mathrm{p}=0.8$.

proposed coding scheme is lower than that of RLNC. For a large network (see Fig. 4), the packet overhead cost of DLNC is similar as that of triangular coding. Even for a large network with a high packet loss probability as shown in Fig. 4, the packet overhead cost of triangular network coding is the same as that of a smaller network with low packet loss probability. In fact the only difference between the results presented in Fig. 3 and Fig. 4 occurs at $M=5$ for triangular network coding, when $\alpha=2$ is required to satisfy inequality 4 , which corresponds to a slightly higher packet overhead cost.

The sudden increase of packet overhead cost for triangular substitution from $M=30$ to $M=35$ and $M=60$ to $M=65$ is explained from the presence of a ceiling logarithm to base two term in the packet overhead cost of our proposed scheme.

\section{CONCLUSiON}

The characteristics of various coding schemes, including triangular coding are summarized in Table I In this work we have proposed an optimal triangular network coding scheme over $G F(2)$, and demonstrated its throughput and computation cost merits over previously proposed network coding schemes. Unlike traditional linear network coding scheme which requires the use of multiplication and Gaussian elimination method at the encoder and decoder respectively, our proposed coding scheme uses XOR addition and backsubstitution method at the encoder and decoder respectively. This way, rather than decreasing the number of computation steps, we decrease the order of computational complexity for both encoding and decoding, without compromising on the throughput performance. Such a coding scheme is throughput optimal, and its packet overhead increases linearithmic with respect to $M$, which can be optimized such that the packet overhead increases linearly with respect to $M$. Most importantly, triangular network coding performance is independent of the packet feedback information. 
TABLE I

SUMMARY OF THE CHARACTERISTICS OF VARIOUS CODING SCHEMES.

\begin{tabular}{|c|c|c|c|c|c|}
\hline & RLNC & DLNC (DGC) [5] & Sparse LNC [19] & Traditional XOR & Triangular Network Coding \\
\hline Throughput & Suboptimal & Optimal & NP-complete & NP-complete & Optimal \\
\hline Algorithm Complexity & $\mathcal{O}\left(M^{2} G\right)$ & $\mathcal{O}\left(N^{2} M^{4}\right)$ & $\mathcal{O}\left(M^{N} N^{2}\right)$ & $\mathcal{O}\left(M^{2} \log _{2} M\right)\lfloor[15]$ & $\mathcal{O}\left(M^{2}\right)$ \\
\hline Encoding Complexity & $\mathcal{O}\left(M^{2} q B\right)$ & $\mathcal{O}\left(M^{2} q B\right)$ & $\mathcal{O}(M q \omega B)$ & $\mathcal{O}(M m B)$ & $\mathcal{O}\left(M^{2} B\right)$ \\
\hline Decoding Complexity & $\mathcal{O}\left(M^{2} q B+M^{3}\right)$ & $\mathcal{O}\left(M^{2} q B+M^{3}\right)$ & $\mathcal{O}\left(M^{2} q B+\omega M^{2}\right)$ & $\mathcal{O}(M m B)$ & $\mathcal{O}\left(M^{2} B\right)$ \\
\hline Packet Overhead (bits) & $M q$ & $M\left|\log _{2} N\right|$ & $M\left|\log _{2} N\right|$ & $M$ & $M+M\left|\log _{2} M\right|$ \\
\hline Packet Feedback & Not required & Required & Required & Required & Not required \\
\hline
\end{tabular}

In addition, apart from the practical implementation significance of our coding scheme, such results are also of interest from an information theoretic studies perspective, as unlike previous works, we have shown that it is possible to obtain optimal coding solution over $G F(2)$ by adding few redundant bits in the packets.

Such a coding scheme would in particular be of interest in energy and processor constraint devices. For our future work, we would like to study the feasibility of extending our current coding scheme for a distributed multi-hop wireless network.

\section{REFERENCES}

[1] Z. Bar-Yossef, Y. Birk, T. S. Jayram, and T. Kol, "Index Coding with Side Information ", in IEEE FOCS, Berkeley, USA, October 2006.

[2] M. Chaudhry and A. Sprintson, "Efficient Algorithms for Index Coding," in IEEE INFOCOM Workshop, Phoeniz, USA, April 2008.

[3] S. Katti, H. Rahul, W. Hu, D. Katabi, M. Medard, and J. Crowcroft, "XORs in the Air: Practical Wireless Network Coding," in ACM SIGCOMM, Pisa, Italy, September 2006.

[4] Y. E. Sagduyu and A. Ephremides, "On Network Coding for Stable Multicast Communication," in IEEE MILCOM, Florida, USA, October 2007.

[5] K. Chi, X. Jiang, and S. Horiguchi, "Network coding-based reliable multicast in wireless networks," Elsevier Computer Networks, vol. 54, no. 11, pp. 1823-1836, August 2010.

[6] J. Heide, M. V. Pedersen, F. H. P. Fitzek, and T. Larsen, "Cautious View on Network Coding - From Theory to Practice," Journal of Communications and Networks, vol. 10, no. 4, pp. 403-411, December 2008.

[7] H. Y. Kwan, K. W. Shum, and C. W. Sung, "Generation of Innovative and Sparse Encoding Vectors for Broadcast Systems with Feedback," in IEEE ISIT, Saint Petersburg, Russia, July 2011.

[8] S. E. Rouayheb, M. Chaudhry, and A. Sprintson, "On the Minimum Number of Transmissions in Single-Hop Wireless Coding Networks," in IEEE ITW, California, USA, September 2007.

[9] J. Heide, M. V. Pedersen, F. H. P. Fitzek, and M. Medard, "On the Code Parameters and Coding Vector Representation for Practical RLNC," in IEEE ICC, Kyoto, Japan, June 2011.

[10] N. Vallina-Rodriguez, P. Hui, J. Crowcroft, and A. Rice, "Exhausting Battery Statistics," in ACM MobiHeld, New Delhi, India, August 2010.

[11] A. Fehske, G. Fettweis, J. Malmodin, and G. Biczok, "The Global Footprint of Mobile Communications: The Ecological and Economic Perspective," IEEE Communications Magazine, vol. 49, no. 8, pp. 5562, August 2011.

[12] H. Shojania and B. Li, "Random Network Coding on the iPhone: Fact or Fiction?" in ACM NOSSDAV, New York, USA, June 2009.

[13] P. Vingelmann, P. Zanaty, F. H. P. Fitzek, and H. Charaf, "Implementation of Random Linear Network Coding on OpenGL-enabled Graphics Cards," in IEEE EW, Aalborg, Denmark, May 2009.

[14] P. Vingelmann, M. V. Pedersen, F. H. P. Fitzek, and J. Heide, "Multimedia Distribution using Network Coding on the iPhone Platform," in ACM MCMC, Firenze, Italy, October 2010.

[15] E. Rozner, A. Padmanabha, Y. Mehta, L. Qiu, and M. Jafry, "ER: Efficient Retransmission Scheme For Wireless LANs," in ACM CoNEXT, New York, USA, December 2007.

[16] H. Shojania and B. Li, "Parallelized Progressive Network Coding With Hardware Acceleration," in IEEE IWQOS, Evaston, USA, June 2007.
[17] K. Park, J.-S. Park, and W. W. Ro, "On Improving Parallelized Network Coding with Dynamic Partitioning," IEEE Transactions on Parallel and Distributed Systems, vol. 21, no. 11, pp. 1547-1560, November 2010.

[18] G. A. Darmohray and E. D. Brooks, "Gaussian Techniques on Shared Memory Multiprocessor Computers," in SIAM PPSC, Los Angeles, USA, December 1987.

[19] C. W. Sung, K. W. Shum, and H. Y. Kwan, "On the Sparsity of a Linear Network Code for Broadcast Systems with Feedback ," in IEEE NetCod, Beijing, China, July 2011.

[20] Y. Li, E. Soljanin, and P. Spasojevic, "Effects of the Generation Size and Overlap on Throughput and Complexity in Randomized Linear Network Coding ", IEEE Transactions on Information Theory, vol. 57, no. 2, pp. 1111-1123, February 2011.

[21] J. Heide, M. V. Pedersen, F. H. P. Fitzek, and T. Larsen, "Network Coding for Mobile Devices - Systematic Binary Random Rateless Codes," in IEEE ICC Workshop, Dresden, Germany, June 2009.

[22] D. Nguyen, T. Tran, T. Nguyen, and B. Bose, "Wireless Broadcast Using Network Coding," IEEE Transactions on Vehicular Technology, vol. 58 , no. 2, pp. 914-925, February 2009.

[23] J. B. Fraleigh and R. A. Beauregard, Linear Algebra. Addison-Wesley Publishing Company, 1987, p 91-97.

[24] A. Menezes, P. Oorschot, and S. Vanstone, Handbook of Applied Cryptography. CRC Press, 1996, p 66. 